\title{
CATALYST CHARACTERIZATION: MÖSSBAUER SPECTROSCOPY IN RELATION TO OTHER TECHNIQUES *
}

\author{
J.W. NIEMANTSVERDRIET \\ Laboratory of Inorganic Chemistry and Catalysis, Eindhoven University of Technology, \\ $5600 \mathrm{MB}$ Eindhoven, The Netherlands
}

For the purpose of catalyst characterization. Mössbauer spectroscopy has matured into a routinely used tool, which is most effective when used in combination with other spectroscopies in a problem-oriented research strategy.

\section{Introduction}

Catalyst characterization plays an important role in catalysis. No less than $78 \%$ out of the about 250 papers presented at the recently held Ninth International Congress on Catalysis (Calgary, Canada, 1988) contained at least some results on the catalyst $(s)$ obtained by characterization techniques (see Fig. 1). The remaining $22 \%$ of the papers dealt with reactions over essentially uncharacterized catalysts $[1,2]$.

Catalyst characterization represents a very active area of research. A recent literature survey identified more than 4000 papers on catalyst characterization over a period of two years [3]. A clearly recognizeable trend in catalysis is that characterization by means of modern spectroscopic techniques is more and more

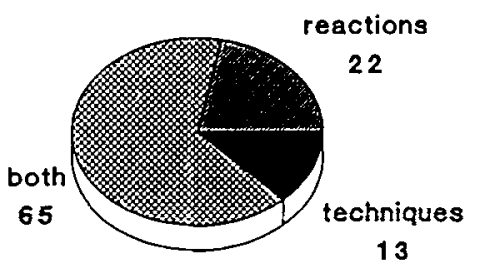

\% Papers 9th Int. Congr. Catalysis

Fig. 1. Percentage of the 250 papers presented at the 9th International Congress on Catalysis (Calgary, Canada, 1988) that dealt with 1) catalytic reactions only, 2) characterization by means of spectroscopic techniques, and 3) a combination of reaction studies with catalyst characterization with one or more techniques.

* Extended abstract.

(C) J.C. Baltzer A.G. Scientific Publishing Company 


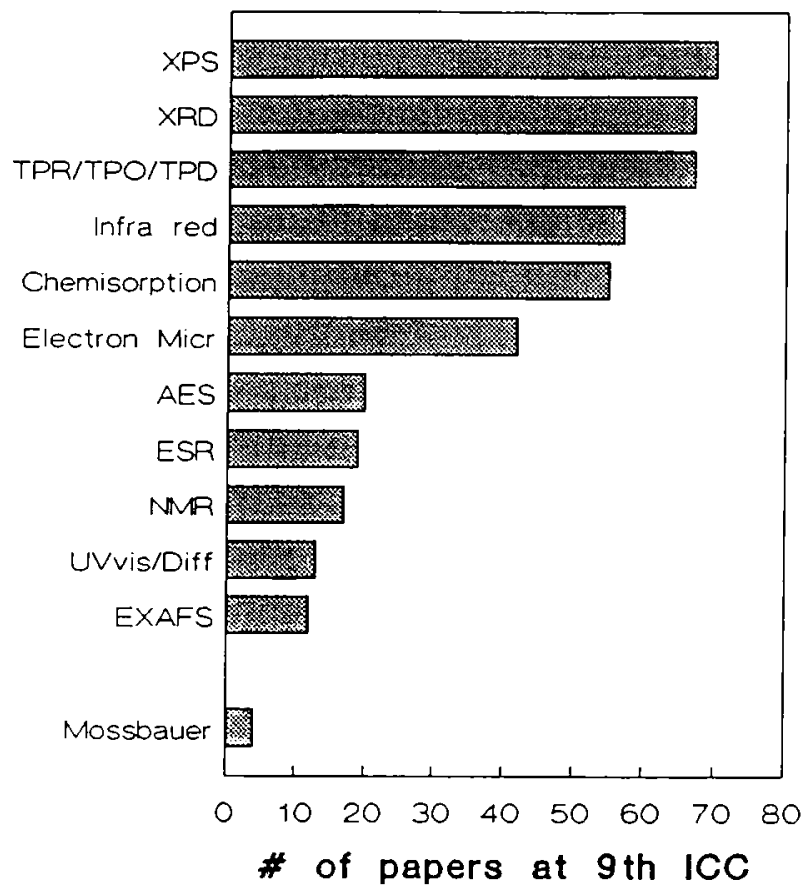

Fig. 2. Bar diagram showing how often certain techniques have been used in the about 250 papers at the 9th International Congress on Catalysis. Mössbauer spectroscopy was used in 4 papers. XPS in 70.

integrated in catalytic investigations as an obvious ingredient of research strategies [4].

As Fig. 2 shows, the most frequently used spectroscopies in catalyst characterization are X-ray photoelectron spectroscopy (XPS). X-ray diffraction (XRD), temperature-programmed reduction, oxidation and desorption (TPR, TPO, TPD), infrared spectroscopy and electron microscopy [2]. Mössbauer spectroscopy is clearly among the incidentally applied techniques in catalysis, but has nevertheless gained general recognition as a valuable tool for specific applications.

\section{Aim of catalyst characterization: fundamental versus applied catalysis}

At present, many workers in catalysis agree that the catalytic properties of a surface are determined by its composition and structure on the atomic scale. This implies that in order to understand catalysis on a fundamental level, one needs to know the exact location of each atom on the surface.

Thus, from a fundamental point of view the ultimate goal of catalyst characterization should be to look at the surface atom by atom. Moreover, as the state of a surface may critically depend on its pretreatment and on the gas atmosphere 
above it, a further requirement would be that the characterization is carried out under reaction conditions (in situ). At present, however, no technique or combination of techniques can achieve the atom by atom investigation of a surface under in situ conditions which would be ideal for fundamental research in catalysis.

The industrial point of view on catalyst characterization is different. Here the main interest is to develop an active, selective, stable and mechanically robust catalyst. In principle, all information that helps to achieve these goals is welcome. For example, establishing empirical relations between the factors that govern catalyst composition, particle size and shape, pore dimensions on one side, and catalytic performance on the other can be extremely useful in the process of catalyst development. Simplifying, one could say that catalyst characterization in industrial research deals with the materials science of catalysts on a more or less microscopic scale, whereas the ultimate goal of fundamental catalytic research is to characterize the surface of a working catalyst on the atomic scale.

\section{Techniques and approaches}

In principle, two approaches can be adopted for fundamental investigations of the relations between catalytic properties on one hand, and catalyst composition and structure on the other. The first is to model the catalytic surface, for example with that of a single crystal. By using the appropriate combination of surface spectroscopies, the desired characterization on the atomic scale is certainly possible in favourable cases. The disadvantage, however, is that although one may be able to study the catalytic properties of such samples under realistic conditions (pressures of $1 \mathrm{~atm}$ or higher), most of the characterization involves electron and ion spectroscopies, which require that the sample is under ultra high vacuum.

The second approach is to study catalysts with in situ techniques such as Infrared and Mössbauer spectroscopy. EXAFS, ESR, NMR and XRD, under reaction conditions, or, as is more often done, under a controlled environment after quenching of the reaction. The in situ techniques, however, are not sufficiently surface specific to yield the desired atom by atom characterization of the surface. They give average properties of the catalyst. When, as often occurs, a catalyst contains several different active sites, all one can do is set up relations between the sum of the catalytic properties and the averaged structure and composition of the catalyst.

\section{Mössbauer spectroscopy in catalyst characterization}

In the last twenty years, Mössbauer spectroscopy has given relevant information on a number of important catalyst systems. Examples are the iron catalysts for ammonia and Fischer-Tropsch synthesis, cobalt-containing hydrotreating 
Table 1

Applications of Mössbauer spectroscopy in catalyst characterization along with the most important alternative techniques (Examples in ref. [2])

\begin{tabular}{ll}
\hline application & most common alternative \\
\hline phase identification & X-ray diffraction (XRD) \\
determination of oxidation state & X-ray photoelectron spectroscopy (XPS) \\
structure information & Extended X-ray Absorption Fine \\
& Structure (EXAFS) \\
particle size determination & XRD, electron microscopy, \\
& chemisorption. magnetic methods \\
kinetics of bulk transformations & gravimetric or magnetic analysis \\
\hline
\end{tabular}

catalysts, supported bimetallic catalysts, and iron in zeolites, see references $[2,5,6]$ for recent reviews. Most applications of Mössbauer spectroscopy to catalysts fall in one of the five categories listed in table 1. The great advantage of Mössbauer spectroscopy is that it can give this information on real catalysts under in situ conditions. Examples of each type of application have been given in recent review [2].

Mössbauer spectroscopy has one important limitation in common with most of the other in situ techniques: the information which is most desirable from a fundamental point of view, namely the atomic composition and structure of the catalytically active surface cannot readily be obtained. It is good to emphasize that the single ideal technique for catalyst characterization does not exist and probably will not exist in the future as well. It is therefore a good strategy to investigate catalysts with a combination of techniques.

With respect to catalytic applications, Mössbauer spectroscopy has matured into a useful tool for which the possibilities and limitations are largely known. In this situation, the technique-oriented approach - i.e. take your favourite spectroscopy and see what it reveals - is unlikely to contribute substantially to new insights in catalysis. The prefered strategy for catalyst characterization would be to take the problem-oriented approach: Identify important questions about a particular catalyst and apply the most appropriate combination of spectroscopies. Such an approach will be particularly effective when close cooperation between experts in catalysis and experts on spectroscopies is possible.

\section{References}

[1] Proc. 9th Int. Congr. on Catalysis, Calgary 1988, ed. M.J. Phillips and M. Ternan (The Chemical Institute of Canada, Ottawa, 1988).

[2] J.W. Niemantsverdriet, Hyp. Int. 47 (1989) 219.

[3] R.L. Austermann, D.R. Denley, D.W. Hart, P.B. Himelfarb. R.M. Irwin, M. Narayana, R. Szentimay, S.C. Tang and R.C. Yeates, Anal. Chem. 59 (1987) 68R.

[4] G.A. Mills, in ref. [1], Vol. 5, p 11.

[5] H. Topsøe, B.S. Clausen and S. Mørup, Hyp. Int. 27 (1986) 231.

[6] A.M. van der Kraan, Hyp. Int. 40 (1988) 211. 